\title{
Unseen and Stressed? Gender Differences in Parent and Teacher Ratings of ADHD Symptoms and Associations With Perceived Stress in Children With ADHD
}

Journal of Attention Disorders I-5

(C) The Author(s) 2016 Reprints and permissions: sagepub.com/journalsPermissions.nav DOI: 10.1 | $77 / 10870547 \mid 665838$ | jad.sagepub.com (S) SAGE

\author{
Johan Isaksson ${ }^{1,2}$, Vladislav Ruchkin ${ }^{1,3,4}$, and Frank Lindblad'
}

\begin{abstract}
Objective: In this study, we investigate the differences between parent and teacher ADHD ratings, and how these ratings relate to perceived stress in children with ADHD. Method: Ratings by parents and teachers with the Swanson, Nolan and Pelham ADHD symptom rating scale (SNAP-IV) were collected from children with a clinical diagnosis of ADHD ( $n$ $=137)$. Also, information on medication was collected. Children ( $\geq 1$ I years of age; $n=64$ ) were invited to complete the Pressure-Activation-Stress scale. Results: Among girls, but not boys, teacher ratings were significantly lower than parental ratings on all symptom scales. Lower teacher ratings on hyperactivity symptoms were associated with higher levels of perceived stress. Conclusion: The findings suggest a potential gender bias in ratings among teachers. Underrated, and hence underidentified, ADHD problems in the school setting seem to increase the perception of stress in the sense of pressure for both girls and boys. (J. of Att. Dis. $X X X X ; X X(X) X X-X X)$
\end{abstract}

\section{Keywords}

ADHD, gender differences, perceived stress, symptom ratings

The informant-based rating scales are considered an important component of the functional assessment of child and adolescent behavior problems (Alberto \& Troutman, 2013), and mental health and education professionals routinely utilize such scales for screening, clinical assessment, or evaluations of interventions (Holmbeck et al., 2008). In school settings, teacher reports are among the most frequently used sources of information about children's behavior. Since teachers have the opportunity to observe their students in a range of situations on a daily basis, they can provide unique perspectives on student behavior. Although teachers have been repeatedly described as well suited for reporting on the range of normative and symptomatic behaviors observed in students (Schmitz, Saudino, Plomin, Fulker, \& DeFries, 1996), there is some evidence that their ratings may be subject to bias and especially gender bias.

A meta-analytic review of studies on gender differences in ADHD concluded that teachers rated boys as significantly more impaired than girls in comparison with parental reports (Gershon, 2002). Previous research has also suggested that among children with ADHD, girls are less likely than boys to be referred for further evaluation and treatment, which at least partially may be related to the assessments made by teachers (Rucklidge, 2010). In a general population sample, both parents and teachers rated boys as having higher levels than girls of inattention and hyperactivity symptoms, but this gender difference was more pronounced for teachers, especially regarding hyperactivity/ impulsivity (Ullebo, Posserud, Heiervang, Obel, \& Gillberg, 2012). Sciutto, Nolfi, and Bluhm (2004) have reported similar findings concerning gender bias and hyperactivity, using a fictional written profile (a two pages long academic record, depicting a 7-year-old with significant impairments in school functioning) and presenting varying information about the child's gender and type of symptoms (inattention, hyperactivity, or comorbid aggression) to different groups of study participants who were all teachers. The teachers were more likely to refer boys than girls with hyperactive behavior for clinical services, suggesting that differences in teacher perceptions of boys' and girls' behaviors may contribute to the gender differences in ADHD referrals.

\footnotetext{
'Uppsala University, Sweden

${ }^{2}$ Karolinska Institutet, Stockholm, Sweden

${ }^{3}$ Yale University, New Haven, CT, USA

${ }^{4}$ Säter Psychiatric Clinic, Sweden

Corresponding Author:

Johan Isaksson, Department of Neuroscience, Child and Adolescent Psychiatry Unit, Uppsala University, Uppsala 75185, Sweden.

Email: johan.isaksson@neuro.uu.se
} 
Previous research, both with children (Isaksson, Nilsson, \& Lindblad, 2015) and adults (Bernardi et al., 2012), has demonstrated that the ADHD diagnosis is associated with higher levels of perceived stress, that is, the subjective experience of demands that exceed their ability to cope, including feelings of pressure, perception of physiological arousal, and a sense of reduced control. In a previous study, girls reported higher levels of perceived stress than boys (Isaksson et al., 2015). It has also been reported previously that children with ADHD have lower levels of the stress hormone cortisol, a phenomenon that seems to develop and increase over time (Isaksson, Nilsson, Nyberg, Hogmark, \& Lindblad, 2012). This may relate to the impaired stress tolerance reported in studies on adults with ADHD (Bernardi et al., 2012; Wender, Wolf, \& Wasserstein, 2001).

Given that teachers seem to underrate hyperactivity symptoms in girls, and that symptoms of inattention do not evoke as much attention in the classroom settings as hyperactivity (Rucklidge, 2010), ADHD symptoms in girls may not be observed and correctly assessed in the school setting. If so, their chances to obtain adequate interventions early in life may be reduced. Hypothetically, this may contribute both to the higher referral age and to the higher levels of perceived stress that are characteristic of ADHD in girls: feeling not having time enough, experiencing pressure from inner demands, and feeling helpless (Isaksson et al., 2015). On the basis of this consideration, we set out to investigate potential differences in parent and teacher ratings in a clinical sample of children with ADHD, hypothesizing that (a) teachers would report lower levels of hyperactivity symptoms in girls than parents, and that (b) lower teacher ratings of ADHD symptoms would, in combination with sex, be associated with higher levels of perceived stress in the children.

\section{Method}

\section{Procedure and Participants}

A group of children and adolescents $(n=137,107$ boys $/ 30$ girls; $M$ age 11.8 years, \pm 2.8 ) with the clinical diagnosis of ADHD according to the International Classification of Diseases (ICD-10) were recruited from child psychiatry outpatient units. The design has been described in detail in a previous publication (Isaksson et al., 2015), in which we compared this group of children with healthy comparisons in relation to perceived stress and cortisol levels. The ADHD diagnoses were based on a clinical interview, physi$\mathrm{cal} /$ neurological assessment, and neuropsychological testing by a clinical psychologist, as well as parent and teacher ratings of symptoms on the SNAP-IV (Swanson et al., 2001). Diagnostic information and the SNAP-IV ratings, collected at the time of an established clinical diagnosis of ADHD, were collected from the child's medical record at their clinical child and adolescent psychiatry units. Information on ADHD medication was collected from parents. Considering a lower reliability of child self-report prior to age of 11 (Compas, Connor-Smith, Saltzman, Thomsen, \& Wadsworth, 2001), when the scales may need simplification and rewording to adjust for cognitive ability of the child, a subset of the children/adolescents ( $\geq 11$ years of age; $n=64,50$ boys/14 girls) was invited to complete the Pressure-Activation-Stress (PAS) scale (Isaksson et al., 2015). The study was approved by the regional ethical review board in Uppsala, decision no. 2009/034. Written informed consent of all participants was obtained after the nature of the diagnostic procedures had been fully explained.

\section{Questionnaires}

For parent and teacher ratings of ADHD symptoms, the SNAP-IV scale was used, including nine items for inattention and nine items for hyperactivity/impulsivity, and two control questions, scored on a 4-point scale: 0 for "not at all," 1 for "just a little," 2 for "quite a bit," and 3 for "very much." Previous studies have demonstrated acceptable internal consistency of the subscales, and a good factor structure (Bussing et al., 2008). The overall Cronbach's alpha varied between .93 and .96 in the parent and teacher ratings.

The PAS scale focuses on two stress dimensions: "pressure" (seven items: too many things at the same time, not time enough, feel under pressure from school demands/ demands at home/inner demands, feel helpless, never feel really free) and "activation" (four items: rush, eat rapidly, keep a high speed all the day, difficult to relax; Lindblad, Backman, \& Akerstedt, 2008). The PAS-items are rated on a 5-point scale (0 for "never," 1 for "rarely," 2 for "sometimes," 3 for "often," and 4 for "always"). The pressure dimension has demonstrated good and the activation dimension acceptable internal consistency. The scale has been shown to discriminate between children with ADHD and healthy comparisons (Isaksson et al., 2015). The overall Cronbach's alpha in the study group was .75.

\section{Statistical Analysis}

All analyses were performed using the Statistical Package for Social Sciences (SPSS-22), a software package commonly used for statistical analysis in several fields like health sciences. As the SNAP-IV-measured symptoms were normally distributed, comparisons between boys and girls on ADHD symptoms were made using the independentsample $t$ test. Gender comparisons of the prevalence of ADHD medication were made using $\chi^{2}$. Individual comparisons of parent and teacher ratings were made using the paired-sample $t$ test. As the data on perceived stress ratings were skewed, we used log-transformation of the ratings in 
Table I. Parental and Teacher Ratings on the SNAP-IV Scale and the Pressure-Activation-Stress (PAS) Scale in the Study Group ( $n=137)$.

\begin{tabular}{|c|c|c|c|c|}
\hline & \multicolumn{2}{|c|}{ Girls } & \multicolumn{2}{|c|}{ Boys } \\
\hline Diagnosis $(n)$ & \multicolumn{2}{|c|}{$A D H D=24 ; A D D=6$} & \multicolumn{2}{|c|}{$\mathrm{ADHD}=97 ; \mathrm{ADD}=10$} \\
\hline ADHD medication $(n, \%)$ & \multicolumn{2}{|c|}{$27(90 \%)$} & \multicolumn{2}{|c|}{$98(91.6 \%)$} \\
\hline SNAP-IV ratings & Parent $M(S D)$ & Teacher $M(S D)$ & Parent $M(S D)$ & Teacher $M(S D)$ \\
\hline Inattention & I.6I (0.68) & $1.04(0.60)$ & $\mathrm{I} .33(0.53)$ & $1.28(0.68)$ \\
\hline Hyperactivity & $1.25(0.76)$ & $0.68(0.79)$ & $1.18(0.70)$ & $\mathrm{I} .0 \mathrm{I}(0.8 \mathrm{I})$ \\
\hline Combined & $\mathrm{I} .44(0.6 \mathrm{I})$ & $0.86(0.63)$ & $1.25(0.53)$ & $1.14(0.67)$ \\
\hline Perceived stress & \multicolumn{2}{|c|}{$M(S D)$} & \multicolumn{2}{|c|}{$M(S D)$} \\
\hline Global score & \multicolumn{2}{|c|}{$22.21(7.2)$} & \multicolumn{2}{|c|}{$17.96(5.8)$} \\
\hline Pressure & \multicolumn{2}{|c|}{ I4.85 (4.5) } & \multicolumn{2}{|c|}{$11.06(4.4)$} \\
\hline Activation & \multicolumn{2}{|c|}{$7.36(3.1)$} & \multicolumn{2}{|c|}{$6.90(2.5)$} \\
\hline
\end{tabular}

Note. $A D D=$ Attention Deficit Disorder; ADHD = Attention Deficit/Hyperactivity Disorder; SNAP-IV = Swanson, Nolan and Pelham ADHD symptom rating scale.

the statistical analyses. Associations between the SNAP-IV ratings and log PAS-scores were assessed using Pearson correlation as well as multiple linear regression. In the regression models, we adjusted for age, sex, and ADHD medication, as these factors may potentially impact the association between SNAP-IV ratings and ratings on perceived stress. Two-tailed tests with $p$ values $<.05$ were considered significant.

\section{Results}

Mean and standard deviations of the SNAP-IV scores and the PAS scores are presented in Table 1. Parental ratings were higher for girls than for boys on inattention $(t=2.34$, $p=.021)$. No sex differences were found in teacher ratings, except for a trend toward higher ratings in boys on hyperactivity and combined symptoms $(p=.085$ and $p=.070$, respectively). There were no differences between boys and girls regarding the prevalence of having ADHD medication. When comparing parent and teacher ratings on the ADHD symptom scale in boys, no differences were found. In girls, parents rated higher than teachers on inattention ( $t=3.47, p=.002$, respectively), on hyperactivity $(t=3.28, p=.004)$, and on combined symptoms $(t=3.63$, $p=.002)$.

Girls reported higher levels of perceived stress on the dimension pressure $(t=2.89, p=.007)$. Parental ADHD symptom ratings were not associated with the stress scores. However, teacher ratings on hyperactivity, but not inattention, were negatively associated with the global score of perceived stress $(r=-.39, p=.008)$ and with the pressure dimension $(r=-.41, p=.005)$, indicating that children with lower teacher rated hyperactivity symptoms perceived higher levels of stress. In linear regression models, adjusted for age, sex, and ADHD medication, the association remained both for the global score $(b=-.36, p=.028)$ and for pressure $(b=-.38, p=.016)$. Age, sex, and ADHD medication were not associated with ratings on perceived stress in the model. Neither were there any interaction effects between sex and teacher ratings on perceived stress.

\section{Discussion}

In this clinical sample of children diagnosed with ADHD, there were differences between teacher and parent ratings for girls, but not boys, with significantly lower teacher ratings on all ADHD symptom scales. Both girls and boys with lower teacher rated hyperactivity reported higher levels of perceived stress.

Our hypothesis that teachers would give lower ratings than parents on hyperactivity symptoms in girls was supported which is in line with previous reports (Gershon, 2002; Sciutto et al., 2004; Ullebo et al., 2012). Similarly, teacher ratings on inattention were lower than parental ratings in girls. These findings may indicate a possible referral bias, where girls are less often than boys referred by teachers for further evaluation and treatment (Gershon, 2002; Rucklidge, 2010; Sciutto et al., 2004). It has been argued that the more common hyperactivity symptoms in boys, with a higher co-morbidity of disruptive behaviors (Gershon, 2002; Taurines et al., 2010), evoke more attention in the classroom situation (Rucklidge, 2010). The finding may also partially explain the previous findings of a higher boy/girl ratio in clinical samples, as compared with the adult population samples (Coles, Slavec, Bernstein, \& Baroni, 2012; Nussbaum, 2012); referrals to psychiatric services during childhood are often initiated by the teachers, while such contacts are often self-initiated in adulthood. Although this study was not designed to evaluate bias in the referral process, the effect of the child's gender on teacher 
ratings is notwithstanding of interest, given that the entire sample had been clinically assessed and diagnosed with ADHD/ADD, and hence the signs of clinically significant impairment were present not only at home but also in the school settings.

Hypothetically, the lower teacher ratings of ADHD symptoms may correspond to an underidentification of ADHD in girls, increasing the risk of not providing the right support or the right form of education which in turn may correspond to the higher ratings of perceived stress in girls with ADHD. Moreover, our results showed that the lower teacher ratings of hyperactivity symptoms in the presence of an ADHD diagnosis were negatively associated with higher stress ratings in our sample, despite of sex. Thus, when the hyperactivity symptoms are less prominent or underidentified in the school setting, the feeling of pressure is higher for both girls and boys. One could also speculate that these children "hold together" during the school-day, meaning that the expressions of hyperactivity are less prominent at school. This may in turn increase the feeling of pressure.

As to limitations, even though we had a considerable number of children with SNAP-IV ratings, only a subset of these children ( $\geq$ age 11 years of age) also completed the PAS scale, thereby decreasing the statistical power. The analyses mainly rely on questionnaire data but the ADHD diagnoses had been validated by structured clinical investigations. For future studies, independent research observations of the child at home and at school could be compared with parent and teacher ratings to clarify the contribution of rating bias.

\section{Acknowledgments}

The authors are grateful to all families who volunteered and to schools and child and adolescent psychiatric units in Uppsala, Enköping, Gävle, and Falun for contributions to the recruitment procedure.

\section{Declaration of Conflicting Interests}

The author(s) declared no potential conflicts of interest with respect to the research, authorship, and/or publication of this article.

\section{Funding}

The author(s) disclosed receipt of the following financial support for the research, authorship, and/or publication of this article: The study was supported by grants from Victoriafonden through the Swedish Brain Foundation (Hjärnfonden), (no specific grant no.). This research was supported by funds from the Uppsala University Hospital Research Fund (ALF) to Johan Isaksson. The research position of Frank Lindblad was financed by the Swedish Council for Working Life and Social Research, grant no. 2006-0197.

\section{References}

Alberto, P., \& Troutman, A. C. (2013). Applied behavior analysis for teachers (9th ed.). Boston, MA: Pearson Education.

Bernardi, S., Faraone, S. V., Cortese, S., Kerridge, B. T., Pallanti, S., Wang, S., \& Blanco, C. (2012). The lifetime impact of attention deficit hyperactivity disorder: Results from the National Epidemiologic Survey on Alcohol and Related Conditions (NESARC). Psychological Medicine, 42, 875-887.

Bussing, R., Fernandez, M., Harwood, M., Wei, H., Garvan, C. W., Eyberg, S. M., \& Swanson, J. M. (2008). Parent and teacher SNAP-IV ratings of attention deficit hyperactivity disorder symptoms: Psychometric properties and normative ratings from a school district sample. Assessment, 15, 317-328.

Coles, E. K., Slavec, J., Bernstein, M., \& Baroni, E. (2012). Exploring the gender gap in referrals for children with ADHD and other disruptive behavior disorders. Journal of Attention Disorders, 16, 101-108.

Compas, B. E., Connor-Smith, J. K., Saltzman, H., Thomsen, A. H., \& Wadsworth, M. E. (2001). Coping with stress during childhood and adolescence: Problems, progress, and potential in theory and research. Psychological Bulletin, 127, 87-127.

Gershon, J. (2002). A meta-analytic review of gender differences in ADHD. Journal of Attention Disorders, 5, 143-154.

Holmbeck, G. N., Thill, A. W., Bachanas, P., Garber, J., Miller, K. B., Abad, M., . . . Zukerman, J. (2008). Evidence-based assessment in pediatric psychology: Measures of psychosocial adjustment and psychopathology. Journal of Pediatric Psychology, 33, 958-980; discussion 981-952.

Isaksson, J., Nilsson, K. W., \& Lindblad, F. (2015). The PressureActivation-Stress Scale in relation to ADHD and cortisol. European Child \& Adolescent Psychiatry, 24, 153-161.

Isaksson, J., Nilsson, K. W., Nyberg, F., Hogmark, A., \& Lindblad, F. (2012). Cortisol levels in children with attention-deficit/ hyperactivity disorder. Journal of Psychiatric Research, 46, 1398-1405.

Lindblad, F., Backman, L., \& Akerstedt, T. (2008). Immigrant girls perceive less stress. Acta Paediatrica, 97, 889-893.

Nussbaum, N. L. (2012). ADHD and female specific concerns: A review of the literature and clinical implications. Journal of Attention Disorders, 16, 87-100.

Rucklidge, J. J. (2010). Gender differences in attention-deficit/ hyperactivity disorder. Psychiatric Clinics of North America, 33, 357-373.

Schmitz, S., Saudino, K. J., Plomin, R., Fulker, D. W., \& DeFries, J. C. (1996). Genetic and environmental influences on temperament in middle childhood: Analyses of teacher and tester ratings. Child Development, 67, 409-422.

Sciutto, M. J., Nolfi, C. J., \& Bluhm, C. (2004). Effects of child gender and symptom type on referrals for ADHD by elementary school teachers. Journal of Emotional and Behavioral Disorders, 12, 247-253.

Swanson, J. M., Kraemer, H. C., Hinshaw, S. P., Arnold, L. E., Conners, C. K., Abikoff, H. B., . . . Wu, M. (2001). Clinical relevance of the primary findings of the MTA: Success rates based on severity of ADHD and ODD symptoms at the end of treatment. Journal of the American Academy of Child and Adolescent Psychiatry, 40, 168-179. 
Taurines, R., Schmitt, J., Renner, T., Conner, A. C., Warnke, A., \& Romanos, M. (2010). Developmental comorbidity in attention-deficit/hyperactivity disorder. Attention Deficit and Hyperactivity Disorders, 2, 267-289.

Ullebo, A. K., Posserud, M. B., Heiervang, E., Obel, C., \& Gillberg, C. (2012). Prevalence of the ADHD phenotype in 7- to 9-year-old children: Effects of informant, gender and non-participation. Social Psychiatry and Psychiatric Epidemiology, 47, 763-769.

Wender, P. H., Wolf, L. E., \& Wasserstein, J. (2001). Adults with ADHD: An overview. Annals of the New York Academy of Science, 931, 1-16.

\section{Author Biographies}

Johan Isaksson, $\mathrm{PhD}$, is a licensed psychologist at the Department of Neuroscience, Child and Adolescent Psychiatry Unit, Uppsala
University, and affiliated at the Department of Women's and Children's Health, Pediatric Neuropsychiatry Unit, Center for Neurodevelopmental Disorders at Karolinska Institutet (KIND), Karolinska Institutet. His research interests include neuropsychiatric and stress-related disorders.

Vladislav Ruchkin, $\mathrm{MD}, \mathrm{PhD}$, is an associate professor at the Department of Child Psychiatry, Uppsala University, Sweden; an assistant professor adjunct at the Yale Child Study Center; and psychiatrist at the Forensic Psychiatric Clinic Sater, Sweden. His research interests include child and adolescent psychopathology and epidemiologic studies of youth.

Frank Lindblad, $\mathrm{MD}, \mathrm{PhD}$, is professor emeritus at the Department of Neuroscience, Child and Adolescent Psychiatry Unit, Uppsala University. He received his $\mathrm{PhD}$ at Karolinska Institutet, Stockholm, Sweden. His main research interests are trauma, stress, and vulnerability. 